plus ou moins faibles en tout cas ; et là, on ignore ce qui se passe exactement, $\mathrm{HCl}$ faisant certainement, entre les divers radicaux aminés de la caséine, une sorte de discrimination, dont le résultat final dépend précisément aussi des conditions de l'opération $(p \mathrm{H}$, température, concentration des divers produits en présence, etc...), ce qui peut sans doute, dans bien des cas, expliquer les différences de résultats obtenus.

Ce nous parait être, en particulier, le cas des différences entre les résultats de Linderstrom-Lang, Cherbuliez et Warner d'une part, GRон d'autre part, en ce qui concerne la caséine $\gamma$, ainsi que le fait qu'il n'est pas absolument certain que les diverses caséines qualifiées $\alpha, \beta, \gamma$ obtenues par les différents auteurs, soient exactement les mêmes corps.

Ajoutons à cela que l'action d'un acide fort comme $\mathrm{HCl}$ est toujours plus ou moins brutale, étant donné qu'il s'agit là d'un produit chimique violent et extrêmement ionisé.

La conclusion est qu'il parait nécessaire de trouver des méthodes d'étude "plus douces») et, pour cela, d'abandonner les traitements chimiques, avec acides forts et bases fortes; on pourrait déjà utiliser des acides organiques beaucoup plus faibles, telles que l'acide acétique et des bases minérales, telles que l'ammoniaque, voire des sels ou des corps organiques neutres $\left(\mathrm{NH}^{4} \mathrm{Cl}\right.$, urée, etc...), comme la chose a été faite par CHERBULIEz et GroH.

Mais sans doute est-il préférable de remplacer ces traitements chimiques par des traitements physiques ou même, simplement mécaniques. C'est du reste ce qui a été fait ; nous en ferons l'objet d'un article subséquent (1).

\title{
INFLUENCE DU CAILLAGE SUR L'UTILISATION DIGESTIVE ET SUR LA VALEUR BIOLOGIQUE DES PROTIDES DU LAIT. \\ ESSAIS DE SUPPLÉMENTATION DES PROTIDES DU LAIT, DU CAILLÉ ET DU CAMEMBERT PAR LA CYSTINE (2)
}

\author{
par \\ Mme LUCIE RANDOIN, Mme Colette JOURDAN \\ et M. JEAN CAUSERET
}

$\mathrm{Au}$ cours de recherches antérieures (3), nous avons montré

(1) M. G. Mocquot, qui a bion vo ulu revoir le prés ant travail, nous a donné quelques judicieux conseils, dont nous 1 a remereions vivement.

(2) Comptes rendus Acad, Agr., 8, 304, 1954.

(3) L. Randoin et C. Jourdan. Comptes rendus Acad. Agr., 38, 101, 1952. L. Rantoin, C. Jourdan et $J$. Causeret. Annales Technol. (I.N.R.A.), ì paraître, 1955. 
que les protides de la plupart des fromages ont un coefficient d'utilisation digestive supérieur à celui des protides du lait, mais que leur valeur biologique est moins élevée que celle de ces derniers.

Il nous a paru intéressant de rechercher à quel stade de la fabrication des fromages se produisent ces modifieations. Nos premières recherches effectuées dans ce sens ont été consacrées à l'influence du caillage du lait sur l'utilisation digestive et sur la valeur biologique de ses constituants azotés. En même temps, nous avons effectué quelques essais de supplémentation du lait, du caillé et du Camembert par la cystine, afin de voir si cet acide aminé - qui constitue avec la méthionine le premier facteur limitant des protides du lait - est capable de relever la valeur biologique des protides des dérivés de ce dernier.

Technique. Nos recherches ont porté sur 3 produits :

- Un lait pasteurisé du eommerce (en carafes);

- Un caillé préparé à partir de ce lait, puis égoutté jusqu'à obtention de 01,500 de lactosérum par litre de lait traité;

- Un caillé préparé de la même manière, puis réadditionné du lactosérum recueilli.

Le caillage du lait a été réalisé de la manière suivante: acidification du lait par addition de $2 \%$ d'un levain de ferments lactiques préparé à partir d'une souche sélectionnée (1); repos à $18^{\circ} \mathrm{C}$. jusqu'à ce que l'acidité du lait atteigne $25^{\circ} \mathrm{D}$. (ce qui demande environ 2 heures); emprésurage à $28^{\circ} \mathrm{C}$. avec de la présure au $1 / 10.000^{\mathrm{e}}$ employée à la dose de $0 \mathrm{gr}$. 15 par litre; repos de 5 heures à $25^{\circ} \mathrm{C}$.; égouttage spontané (environ 1 heure).

Chacun des produits à comparer a été introduit dans un mélange alimentaire, à des doses telles que les régimes obtenus (régimes $\mathrm{I}$, II et III) contiennent $10 \%$ de protides, Deux autres régimes (régimes IV et V) ont été préparés en associant au même mélange, deux Camemberts du commerce de marques différentes, ces deux régimes renfermant comme les précédents $10 \%$ de protides.

Les régimes ainsi préparés avaient la composition indiquée à la page suivante (les quantités indiquées correspondent à 100 grammes de matière sèche au total).

Par ailleurs, einq autres régimes (I bis à $\mathrm{V}$ bis) ont été préparés à partir des régimes $\mathrm{I}$ à $\mathrm{V}$ par substitution de $0 \mathrm{gr}$. 2 de eystine (pour 100 grammes de matière sèche) à une égale quantité d'amidon (soit un supplément de 2 grammes de cystine pour 100 grammes de protides).

(1) En prenant comme milieu, 100 grammes de lait reconstitué à partir de 9 grammes de lait en poudre, et 91 grammes d'eau de ville et stérilisé à l'autoclave à $118^{\circ} \mathrm{C}$. pendant vingt minutes. 


$\begin{array}{ccccc}\text { Rég. I } & \text { Rég. II } & \text { Rég. III } & \text { Rég. IV } & \text { Rég. V } \\ 35 & 35 & 35 & 38 & 39,5 \\ 35 & 35 & 35 & 30 & 30 \\ - & - & - & 3 & 3 \\ 0,075 & 0,075 & 0,075 & 0,075 & 0,075 \\ - & - & - & 2 & 2 \\ 300 & - & - & - & - \\ - & 150 & 150 & - & - \\ - & - & 150 & - & - \\ - & - & - & 49 & - \\ - & - & - & - & 43,5\end{array}$

Les expériences ont porté sur 74 rats albinos mâles de 70 à 100 grammes. Durant une première période de 9 jours, ils ont tous été soumis à un même régime sans protéines à base de : amidon, 50 ; saccharose, 32 ; huile d'arachide, 12 ; mélange salin de Hubbel, 4 ; agar-agar, 2 et mélange vitaminique (2).

Du quatrième au neuvième jour, les fèces et l'urine ont été recueillies en vue d'y doser l'azote.

A la fin de cette première période, les 74 animaux ont été répartis en 10 lots de 9 sujets (lots I-I bis, II-II bis, III-III bis) ou 5 sujets (lots IV-IV bis, V-V bis), puis soumis aussitôt aux régimes I-I bis à $\mathrm{V}-\mathrm{V}$ bis, dont la composition a été indiquée plus haut. Cette seconde période expérimentale (période protéique) a duré 16 jours, la récolte des fèces et de l'urine ne commençant que le septième jour.

Le coefficient de digestibilité des protides des produits étudiés a été calculé par la formule de P. LeLiu :

( $\mathrm{N}$ ingéré pér. prot. - $\mathrm{N}$ ing. pér. sans prot.) C. $\mathrm{U} . \mathrm{D} .=\frac{(\mathrm{N} \text { fécal pér. prot. }-\mathrm{N} \text {. fécal pér. sans prot. })}{\mathrm{N} \text { ingéré pér. prot. }-\mathrm{N} \text { ingéré pér. sans prot. }} \times 100$

La valeur biologique des protides a été calculée par la formule de MaRTin et RoBison :

$$
\begin{aligned}
\mathrm{V} . \mathrm{B} . & =\frac{\text { Bilan azoté pér. prot. }- \text { Bilan azoté pér. sans prot. }}{\text { (N ingéré pér. prot. }-\mathrm{N} \text { fécal pér. prot. })-} \times 100 \\
& \text { ( } \mathrm{N} \text { ingéré pér. sans prot. }-\mathrm{N} \text { fécal pér. sans prot. })
\end{aligned}
$$

où le bilan azoté représente la quantité d'azote retenu (caleulée

(1) Iodure de potassium, alun de potassium, sulfate de manganèse, fluorure de sodium et sulfate de cuivr3, dans les mêmes proportions que dans le mélange salin de Hubbel et coll. (Journal Nutr., 14, 273, 1937.

(2) Par kilogramm э sec de régime : thiamine, 2 milligrammes ; riboflavine, 3 milligrammes ; amide nicotinique, 50 milligrammэs; pyridoxins, $2 \mathrm{mgr}$. 5 ; pantothénate de calcium, 10 milligrammวs. 
elle-même par différence entre la quantité ingérée et la somme des quantités éliminées par les fèces et par l'urine).

Résultats. Les résultats obtenus en moyenne sont les suivants :

Lots C.U.D. des protides

I Lait $\ldots \ldots \ldots \ldots \ldots \ldots \ldots \ldots$

II Caillé $\ldots \ldots \ldots \ldots \ldots \ldots \ldots \ldots \ldots$

III Caillé + lactosérum $\ldots \ldots \ldots \ldots \ldots$

IV Cámembert $n^{\circ} 1 \ldots \ldots \ldots \ldots \ldots$

V Camembert $n^{\circ} 2 \ldots \ldots \ldots \ldots$

I bis Lait + eystine ...........

II bis Caillé + eystine ...........

III bis Caillé + lactosérum + cystine.

IV bis Camembert $n^{\circ} \mathbf{1}+$ eystine ....

$\mathrm{V}$ bis Camembert $\mathrm{n}^{\circ} 2+$ eystine ....
91,4

95,0

95,2

95,6

96,6

90,0

93,4

92,7

96,6

96,3
V.B. des protides
84,0
63,9
74,2
58,8
66,1
84,4
62,9
79,4
63,0
66,7

\section{CONCLUSION}

De ces résultats, on peut tirer les conclusions suivantes :

$1^{0}$ L'utilisation digestive des protides du caillé est légèrement supérieure à celle des protides du lait; par contre, leur valeur biologique est nettement abaissée ;

$2^{\circ}$ Les protides du mélange caillé + lactosérum ont un coefficient d'utilisation digestive sensiblement égal à celui des protides du caillé, tandis que leur valeur biologique est intermédiaire entre celle des protides du lait et celle des protides du caillé. En conséquence, il semble que la digestibilité supérieure des protides du caillé soit due au fait même du caillage, alors que l'infériorité de leur valeur biologique résulte tout à la fois du caillage et de l'élimination des protéínes solubles du lait;

$3^{\circ} \mathrm{Du}$ double point de vue de l'utilisation digestive et de la valeur biologique, les protides des deux échantillons de Camembert étudiés "se classent " sur le même plan que ceux du caillé. Bien que les conditions d'obtention de ce dernier ne soient pas absolument identiques à celles des caillés de fromagerie, il apparaît done vraisemblable que la maturation du fromage n'exerce qu'une influence secondaire sur les propriétés biologiques de ses constituants azotés ;

$4^{\circ}$ Malgré la relative pauvreté des protides du lait et de ses dérivés en cystine, l'addition de cet acide aminé au lait, au caillé ou au Camembert n'exerce, dans les conditions de nos expériences, aucun effet de supplémentation biologique.

(Institut national de la Recherche agronomique.) 